\title{
Differential activation of Fyn kinase distinguishes saturated and unsaturated fats in mouse macrophages
}

\author{
Elena Tarabra ${ }^{1}$, Ting-Wen An Lee ${ }^{4}$, Victor A. Zammit' ${ }^{2}$, Manu Vatish ${ }^{3}$, Eijiro Yamada ${ }^{5}$, \\ Jeffrey E. Pessin ${ }^{1}$ and Claire C. Bastie ${ }^{1,2}$ \\ ${ }^{1}$ Department of Medicine and Molecular Pharmacology, Albert Einstein College of Medicine, Bronx, NY, USA \\ ${ }^{2}$ Division of Biomedical Sciences, Warwick Medical School, University of Warwick, Coventry, UK \\ ${ }^{3}$ Nuffield Department of Obstetrics \& Gynaecology, University of Oxford, Oxford, UK \\ ${ }^{4}$ Department of Pediatric Endocrinology, The Valley Hospital, Ridgewood, NJ, USA \\ ${ }^{5}$ Department of Medicine and Molecular Science, Gunma University Graduate School of Medicine, Maebashi, Japan \\ Correspondence to: Claire C. Bastie, email: c.c.bastie@warwick.ac.uk \\ Keywords: Fyn kinase, macrophages, fatty acids, adipose tissue, obesity
}

Received: March 29, $2017 \quad$ Accepted: July 19, $2017 \quad$ Published: September 21, 2017

Copyright: Tarabra et al. This is an open-access article distributed under the terms of the Creative Commons Attribution License 3.0 (CC BY 3.0), which permits unrestricted use, distribution, and reproduction in any medium, provided the original author and source are credited.

\section{ABSTRACT}

Diet-induced obesity is associated with increased adipose tissue activated macrophages. Yet, how macrophages integrate fatty acid (FA) signals remains unclear. We previously demonstrated that Fyn deficiency (fynKO) protects against high fat diet-induced adipose tissue macrophage accumulation. Herein, we show that inflammatory markers and reactive oxygen species are not induced in fynKO bone marrow-derived macrophages exposed to the saturated FA palmitate, suggesting that Fyn regulates macrophage function in response to FA signals. Palmitate activates Fyn and re-localizes Fyn into the nucleus of RAW264.7, J774 and wild-type bone marrowderived macrophages. Similarly, Fyn activity is increased in cells of adipose tissue stromal vascular fraction of high fat-fed control mice, with Fyn protein being located in the nucleus of these cells. We demonstrate that Fyn modulates palmitate-dependent oxidative stress in macrophages. Moreover, Fyn catalytic activity is necessary for its nuclear re-localization and downstream effects, as Fyn pharmacological inhibition abolishes palmitate-induced Fyn nuclear redistribution and palmitate-dependent increase of oxidative stress markers. Importantly, mono-or polyunsaturated FAs do not activate Fyn, and fail to re-localize Fyn to the nucleus. Together these data demonstrate that macrophages integrate nutritional FA signals via a differential activation of Fyn that distinguishes, at least partly, the effects of saturated versus unsaturated fats.

\section{INTRODUCTION}

Obesity, the most potent trigger for the development of type 2 diabetes and insulin resistance, has reached pandemic proportions and will affect more than 475 million individuals worldwide in the next 15 years [1]. Obesity is the result of dysregulation of a number of biological processes, including the disturbance of whole body homeostasis including immune functions [2]. In this regard, obesity is associated with systemic low-grade inflammation, also called meta-inflammation characterized by accumulation of immune cells in peripheral tissues such as liver, skeletal muscle and adipose tissue [3-6]. In addition to the increased immune cell number, obesityinduced inflammation is defined by a modification of immune cell populations with increased B [6] and T lymphocytes [7], neutrophils [8] and mast cells [9]; and decreased natural killer cells and eosinophil numbers, 
promoting insulin resistance, particularly in the adipose tissues of obese individuals [10]. Interestingly, metainflammation shares similar sets of signaling pathways with classical inflammation. However, the hallmark of meta-inflammation is that the inflammatory processes are stimulated by the same excessive nutrient supply that also dysregulates energy metabolism and insulin sensitivity.

Remarkably, macrophages can represent up to $40 \%$ of the adipose tissue cell population in morbidly obese patients [11]; and there is substantial evidence that macrophages are involved in the establishment of inflammation-associated insulin resistance in this tissue. However, it is still under scrutiny whether the sole increase in macrophage number [12] or a switch in their phenotype from anti- to pro-inflammatory $[13,14]$ is responsible for this inflammation. Recently, the presence of macrophages distinct from the M1 pro-inflammatory phenotype and resemble the M2 phenotype have been described in adipose tissues of obese humans and mice [14]. Interestingly, these macrophages display a unique set of markers involved in lipid metabolism and induced by the saturated fatty acid (FA) palmitate, demonstrating that high fat diets induce a change in macrophage phenotype and function.

Determining the molecular mechanisms that trigger these changes under a high fat diet regime is likely to offer insights into the metabolic consequences of obesity. However, how cells "sense" FA cues remains to be clarified. In this regard, recent studies have focused on molecules that could provide a link between FA sensing and immune function. AMP-activated protein kinase (AMPK) has recently being suggested to mediate some of the anti-inflammatory effects of unsaturated FAs in macrophages $[15,16]$ by decreasing pro-inflammatory gene expression $[17,18]$. Recently, the nuclear-factor erythroid 2-related factor $2(\mathrm{Nrf} 2)$, a transcription factor playing physiological roles in oxidative stress has emerged as an important factor in the orchestration of the effects of FAs on macrophage function [19]. Indeed, high fat feeding inhibits Nrf2 downstream targets [20]. Interestingly, Fyn tyrosine kinase phosphorylates Nrf2, promoting Nrf2 nuclear exclusion and subsequent degradation [21-23].

Fyn-a member of the Src family of non-receptor tyrosine kinases- is an important molecule in the integration of metabolic and inflammatory pathways. Its role as a modulator of the innate immune function is well established, and we have previously demonstrated that Fyn is a key regulator of glucose and energy homeostasis [24]. Consistently with the role of Fyn in lipid metabolism, we have demonstrated that fyn-deficient (fynKO) mice display higher rates of long chain FA oxidation, as a result of enhanced LKB1 and AMPK activities in adipose tissue and skeletal muscle [25]. More recently, we reported that Fyn deficiency protects animals from diet-induced insulin resistance despite induction of obesity [26]. Importantly, we showed that the number of macrophages was greatly reduced in the adipose tissue of fynKO animals after a high fat diet [26]. This suggested that Fyn might regulate macrophage function in response to FAs. Therefore, in this report we investigated 1) the role of Fyn kinase in saturated FA-induced macrophage activation and 2) the effects of saturated and unsaturated FAs on Fyn function and downstream signaling.

\section{RESULTS}

\section{Increased Fyn activity in adipose tissue stromal vascular fraction of high fat-fed mice}

High fat diet-fed fynKO mice display reduced systemic inflammation and decreased macrophage
A

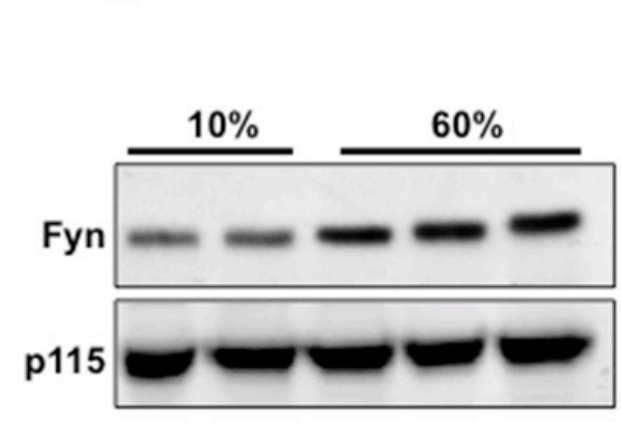

B

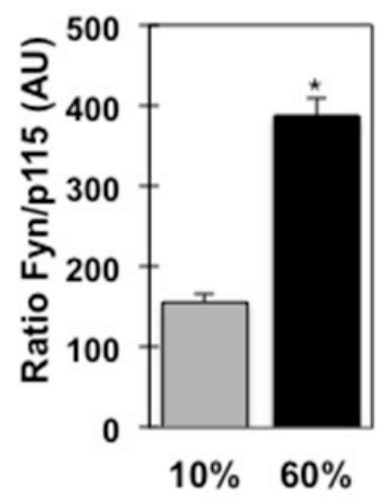

C

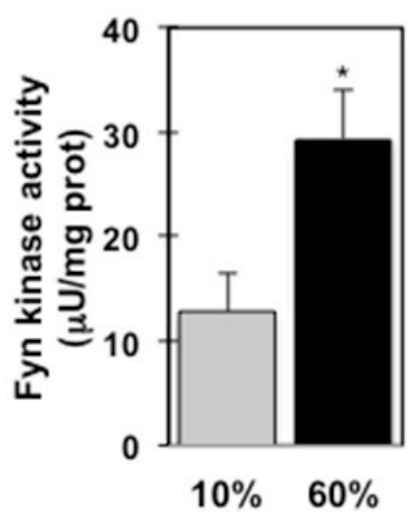

Figure 1: Fyn kinase expression and activity are increased in isolated ATMs and SVF of high fat-fed mice. (A) Fyn protein expression in adipose tissue macrophages (ATMs) from low fat-fed $(10 \% \mathrm{Kcal})$ and high fat-fed $(60 \% \mathrm{Kcal}) \mathrm{mice}(\mathrm{n}=8)$. P115 was used as loading control. (B) Signal quantification of Fyn protein expression normalized by p115. (C) Fyn tyrosine kinase activity in the stromal vascular fraction of adipose tissue of mice fed either a $10 \% \mathrm{Kcal}$ fat diet or a $60 \%$ Kcal fat diet for 8 weeks, ( $\mathrm{n}=4$ experiments), ${ }^{*} p$ $(60 \%$ vs. $10 \%)<0.05$. 
number in the stromal vascular fraction (SVF) of their adipose tissue [26], suggesting that Fyn participates in the deleterious effects of high fat diets on inflammatory processes. Interestingly, Fyn protein expression was significantly increased in isolated adipose tissue macrophages (ATMs) from wild type mice following 8 weeks of high fat diet $(60 \% \mathrm{Kcal})$ (Figure 1A, B). Consistent with this increase, Fyn tyrosine kinase activity was increased more than 2-fold in the SVF of adipose tissue of high fat-fed mice (Figure 1C), suggesting that dietary fats might regulate Fyn activity.

\section{Inflammation markers and ROS are reduced in} palmitate-treated fynKO macrophages

To investigate the mechanisms by which Fyn expression/activity affects macrophage response to FAs, we first assessed inflammatory marker expressions in isolated ATMs from high fat-fed control and fyn $\mathrm{KO}$ mice. Consistent with our previous report [26], TNF $\alpha$ and IL6 expression were reduced in macrophages from high fat-fed fynKO mice (Figure 2A). Importantly, whilst palmitate increased inflammatory markers (NOS2,
A

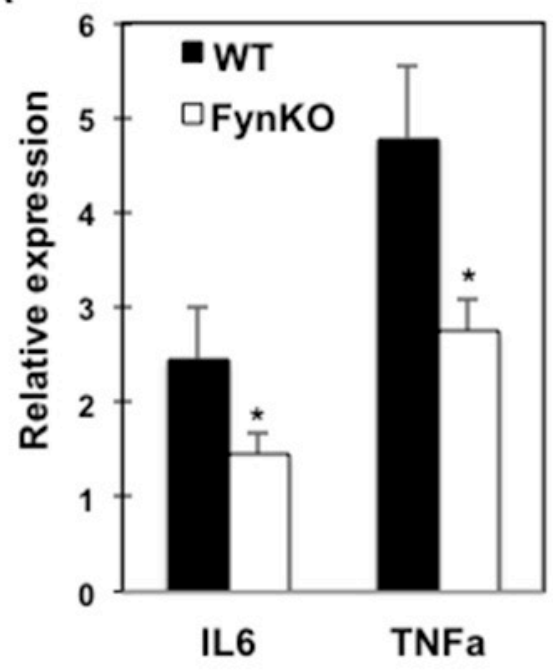

B

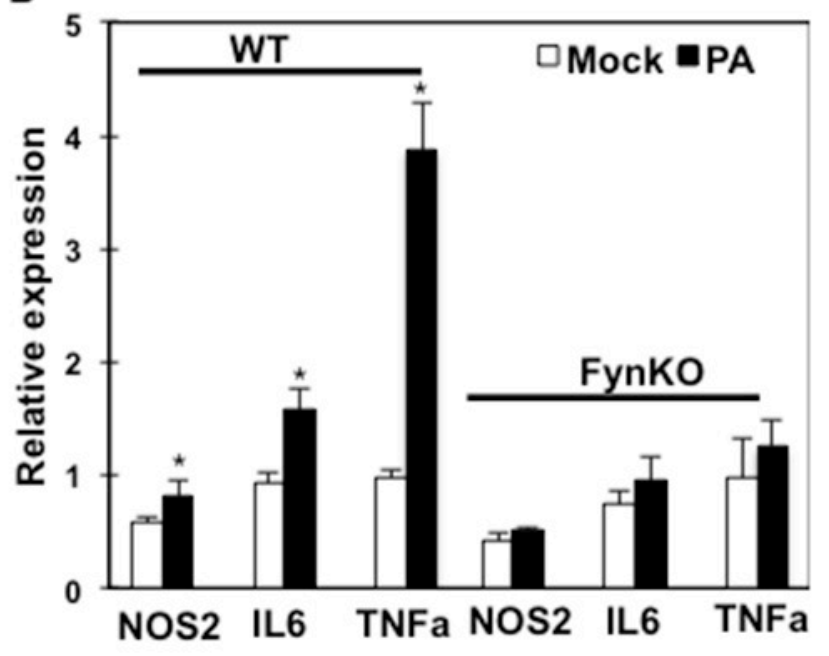

C

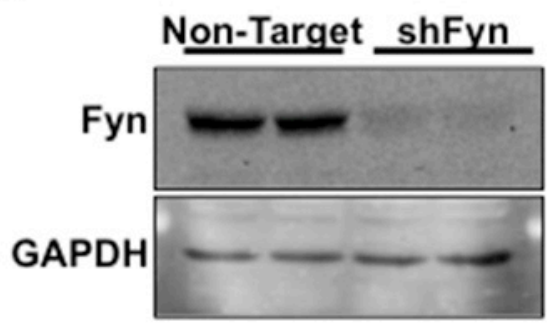

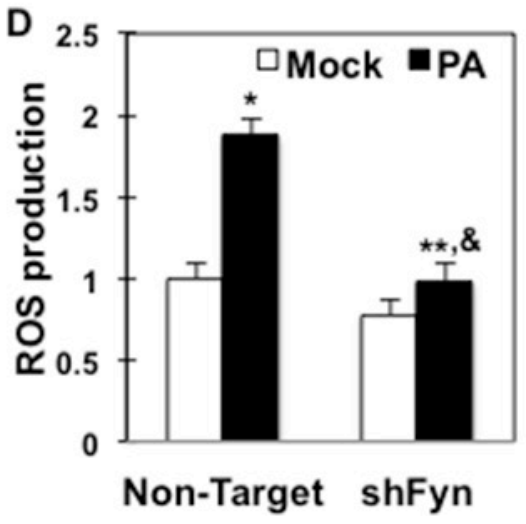

Figure 2: Inflammation markers and ROS production are reduced in Fyn-deficient macrophages. (A) Inflammation markers (IL6, TNF $\alpha$ ) in wild type (WT) and fynKO mice ATMs. (B) Inflammation markers (NOS2, IL6, TNF $\alpha$ ) in bone marrow-derived macrophages (BMDMs) of WT and fynKO mice treated with $150 \mu \mathrm{M}$ palmitate (PA) (dark bars) or BSA alone (Mock) (open bars) $(\mathrm{PA}: \mathrm{BSA}=2)\left(\mathrm{n}=3\right.$ experiments). ${ }^{*} p<0.05$. (C) RAW264.7 macrophages were infected with either shRNA encoding for a non-mammalian target (non-target) or fyn sequences (shFyn). Fyn protein expression was determined by immunoblotting in two separate cell lines. (D) ROS production in shFyn-RAW264.7 cells and control cells (non-target) treated with $150 \mu \mathrm{M}$ PA or with BSA alone (Mock) (PA:BSA=2). Data were normalized to mock in control cells $(\mathrm{n}=4$ experiments), * non-target mock $v s$. non-target PA $p<0.05$; **shFyn mock $v s$. shFyn PA $p<0.05$; \& non-target PA $v s$. shFyn PA $p<0.05$. (E) NAD(P)H oxidase mRNA expression in BMDMs of WT and fynKO mice treated with $150 \mu \mathrm{M}$ PA (dark bars) or with BSA alone (open bars) for $4 \mathrm{~h}(\mathrm{PA}: \mathrm{BSA}=2),(\mathrm{n}=4$ experiments), * WT mock $v s$. WT PA $p<0.05$; ** fynKO mock $v$ s. fynKO PA $p<0.05$, \& WT PA $v$ s. fynKO PA $p<0.05$. 
IL6 and $T N F \alpha$ ) in bone marrow-derived macrophages (BMDMs) from low fat-fed control mice, these markers were not induced in BMDMs of low fat-fed fynKO mice (Figure 2B). Using shRNA-mediated knock down, we silenced fyn in RAW264.7 macrophages (shFynRAW264.7) (Figure 2C). The reactive oxygen species (ROS) production that accompanies the early stage of inflammatory processes was increased in RAW264.7 macrophages after incubation with palmitate, but not in shFyn-RAW264.7 macrophages (Figure 2D). Similarly, $\mathrm{NAD}(\mathrm{P}) \mathrm{H}$ oxidase expression, the enzyme catalyzing the production of superoxide, was not induced in fyn-deficient macrophages after palmitate exposure (Figure 2E). These data suggest that lack of Fyn kinase expression in macrophages is required to lessen the inflammatory effects mediated by saturated FAs.

\section{Saturated but not unsaturated FAs redistribute Fyn in the nucleus of macrophages}

Fyn subcellular localization varies from cytoplasmic to nuclear depending on which signals the cells were exposed to [22, 27]. Interestingly, Fyn protein expression was increased in the adipose tissue

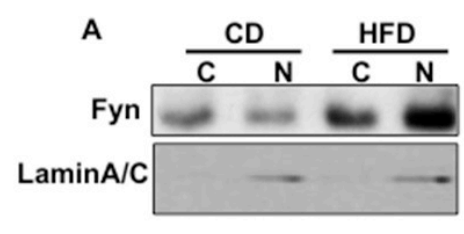

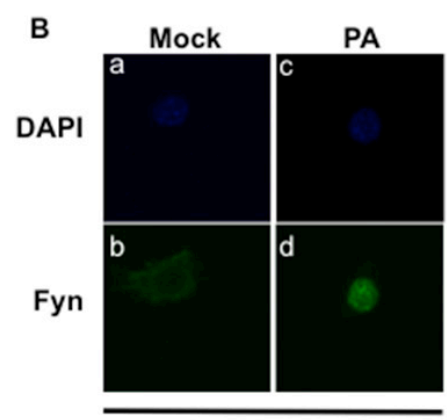

RAW264.7

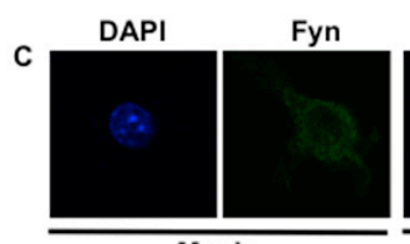

Mock

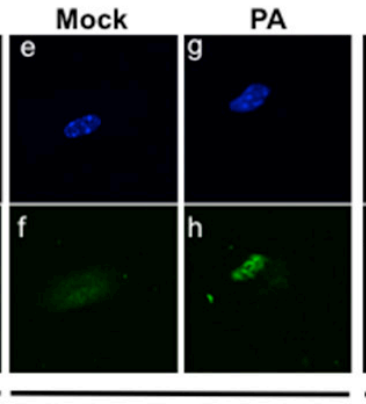

BMDM

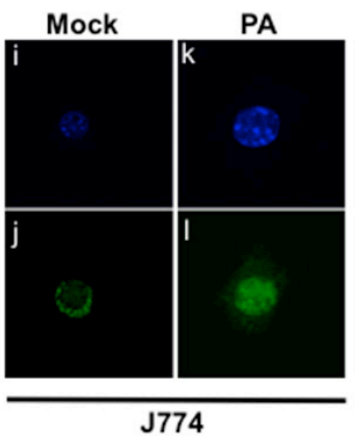

D

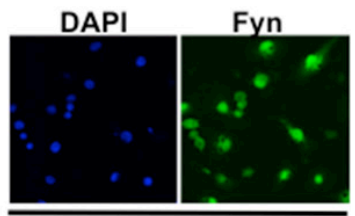

PA:DHA $=2$

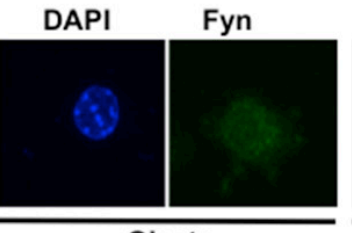

Oleate
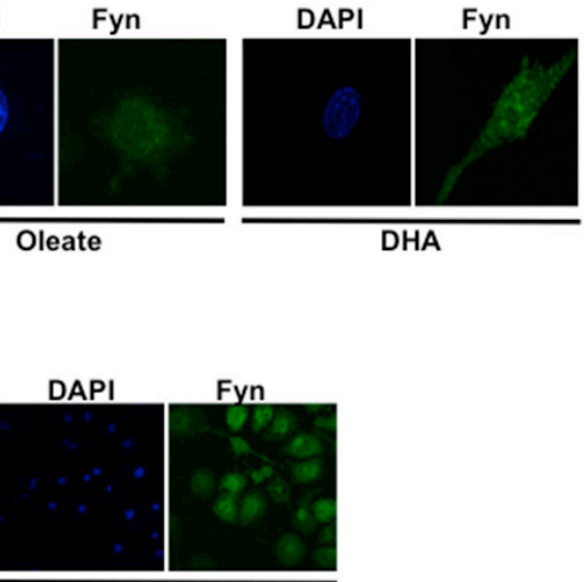

PA:DHA $=0.5$

Figure 3: Palmitate exposure induces Fyn nuclear re-localization. (A) Cellular fractionation of cells from adipose tissue stromal vascular fractions from low fat-fed (CD) and high fat-fed (HFD) control mice. Fyn protein expression was assessed in cytoplasmic and nuclear fractions. (B) RAW264.7 macrophages (a-d), BMDMs from wild type mice (e-h) and J774 macrophages (i-1) were incubated with BSA (Mock) or $150 \mu \mathrm{M}$ palmitate (PA) (FA:BSA=2). Nuclei (a, c; e, g; i, k) were visualized using DAPI staining and Fyn subcellular localization ( $b, d ; f, h ; j, l)$ was assessed by immunofluorescence ( $n=7$ experiments). (C) Fyn subcellular localization in RAW264.7 macrophages incubated with BSA(Mock) or $150 \mu \mathrm{M}$ Oleate or $150 \mu \mathrm{M}$ DHA(FA:BSA=2) for 10 min (n=4 experiments). (D) Fyn subcellular localization in RAW264.7 macrophages incubated with a mixture of palmitate (PA) and DHA at different ratios, ( $\mathrm{n}=3$ experiments). 
SVF of high fat-fed mice (Figure 3A), consistent with increased Fyn activity (Figure 1C). Also, Fyn protein expression was increased in the nuclear fraction of SVF from high fat-fed mice whilst Fyn signal was higher in the cytoplasm fraction of SVF from low fat-fed mice (Figure 3A). Together, these data suggested that high fat-diet re-localized Fyn into the nucleus of cells. Consistent with this, endogenous Fyn was predominantly localized in the cytoplasm of mock (BSA)-stimulated RAW264.7 cells but was re-distributed to the nucleus following palmitate exposure (Figure 3B, panels a-d). Similar results were obtained with wild type BMDMs (Figure 3B, panels e-h) and the J774 macrophage cell line (Figure 3B, panels i-1). The percentage of cells with Fyn signal predominantly in the nucleus in each cell line is presented in Supplementary Figure 1A, B and C.

Interestingly, Fyn re-distribution to the nucleus appeared to be restricted to palmitate treatment, as Fyn remained in the cytoplasm in $70 \%$ of cells treated with the mono-unsaturated FA oleate and in approximately $85 \%$ of cells exposed to the polyunsaturated FA docosahexaenoic acid (DHA) (Figure 3C and Supplementary Figure 2A) or polyunsaturated FA eicosapentaenoic acid (data not shown). In line with this, subcellular fractionation of RAW264.7 cells confirmed that Fyn localized in nuclear fractions after palmitate exposure but localized in the cytoplasm after incubation with DHA (Supplementary Figure 2B). Fyn also localized to the nucleus in $80 \%$ of the cells exposed to a mixture of saturated (palmitate) and unsaturated (DHA) FAs at a ratio similar to that found in high fat diets (PA:DHA ratio = 2) (Figure 3D). But, only $20 \%$ of the cells presented Fyn in the nucleus (Figure $3 \mathrm{D})$ when they were incubated in a mixture where DHA concentration was higher than this of palmitate (PA:DHA ratio $=0.5$ ). These results are essentially consistent with the fact that Fyn protein can be found in the nucleus of
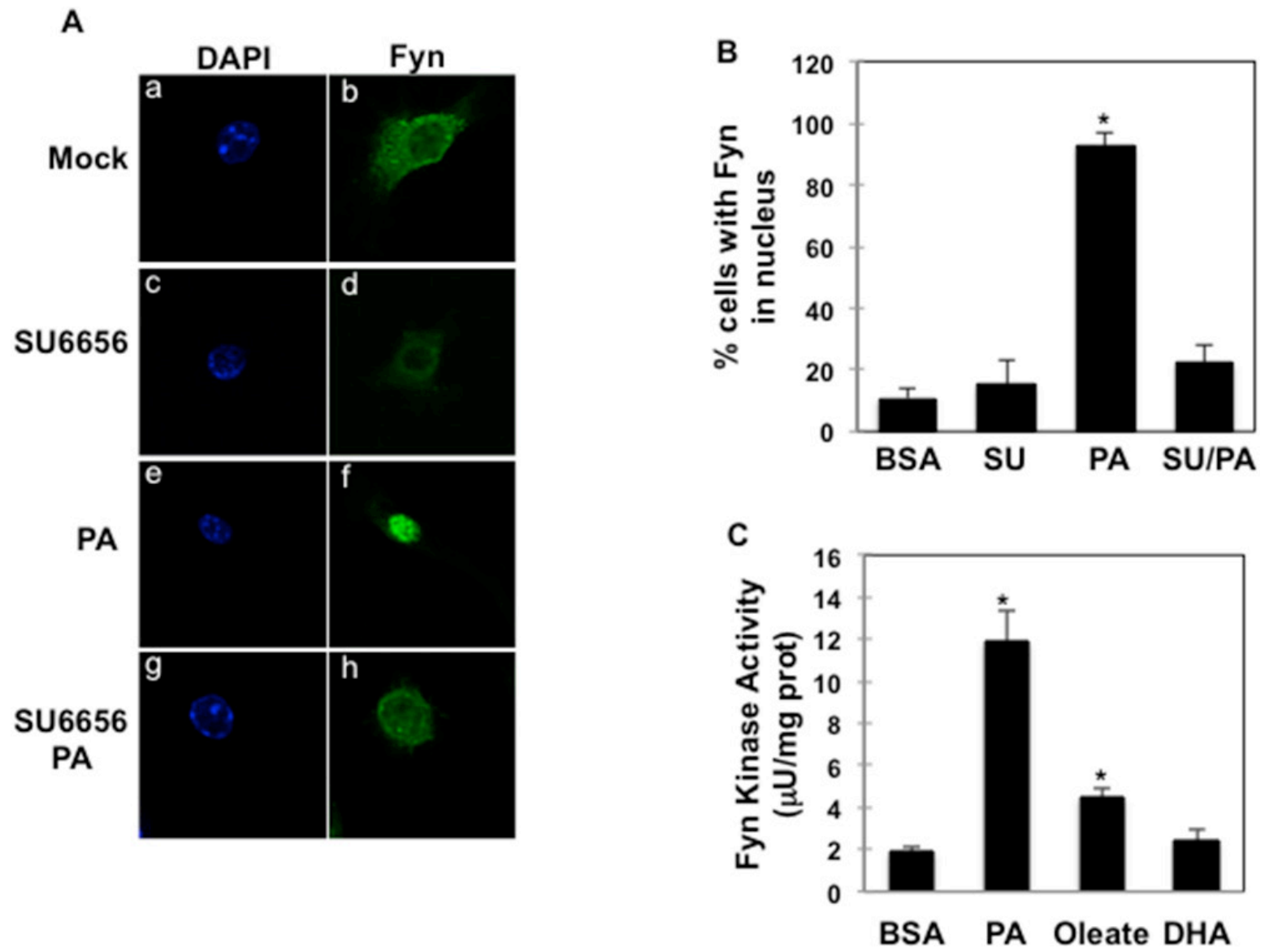

Figure 4: Fyn activity is necessary for palmitate-dependent Fyn nuclear re-localization. (A) RAW264.7 macrophages were incubated with BSA (a, b); $5 \mu \mathrm{M}$ SU6656 (c, d), $150 \mu \mathrm{M}$ palmitate (PA) (e, f) or SU6656 and palmitate (g, h) (PA:BSA=2). Nuclei and Fyn localization were visualized as above, ( $\mathrm{n}=5$ experiments). (B) Percentage of RAW264.7 cells with Fyn signal detected in the nucleus $\left(\mathrm{n}=5\right.$ experiments), ${ }^{*} p$ (PA $v s$. BSA) $<0.05$. (C) RAW264.7 macrophages were incubated with BSA or $150 \mu \mathrm{M}$ Oleate or $150 \mu \mathrm{M}$ DHA $(\mathrm{FA}: \mathrm{BSA}=2)$ for $10 \mathrm{~min}$. Fyn kinase activity was assessed as described in Material and Methods $(\mathrm{n}=4$ experiments $),{ }^{*} p(\mathrm{FA} v s . \mathrm{BSA})<0.05$. 

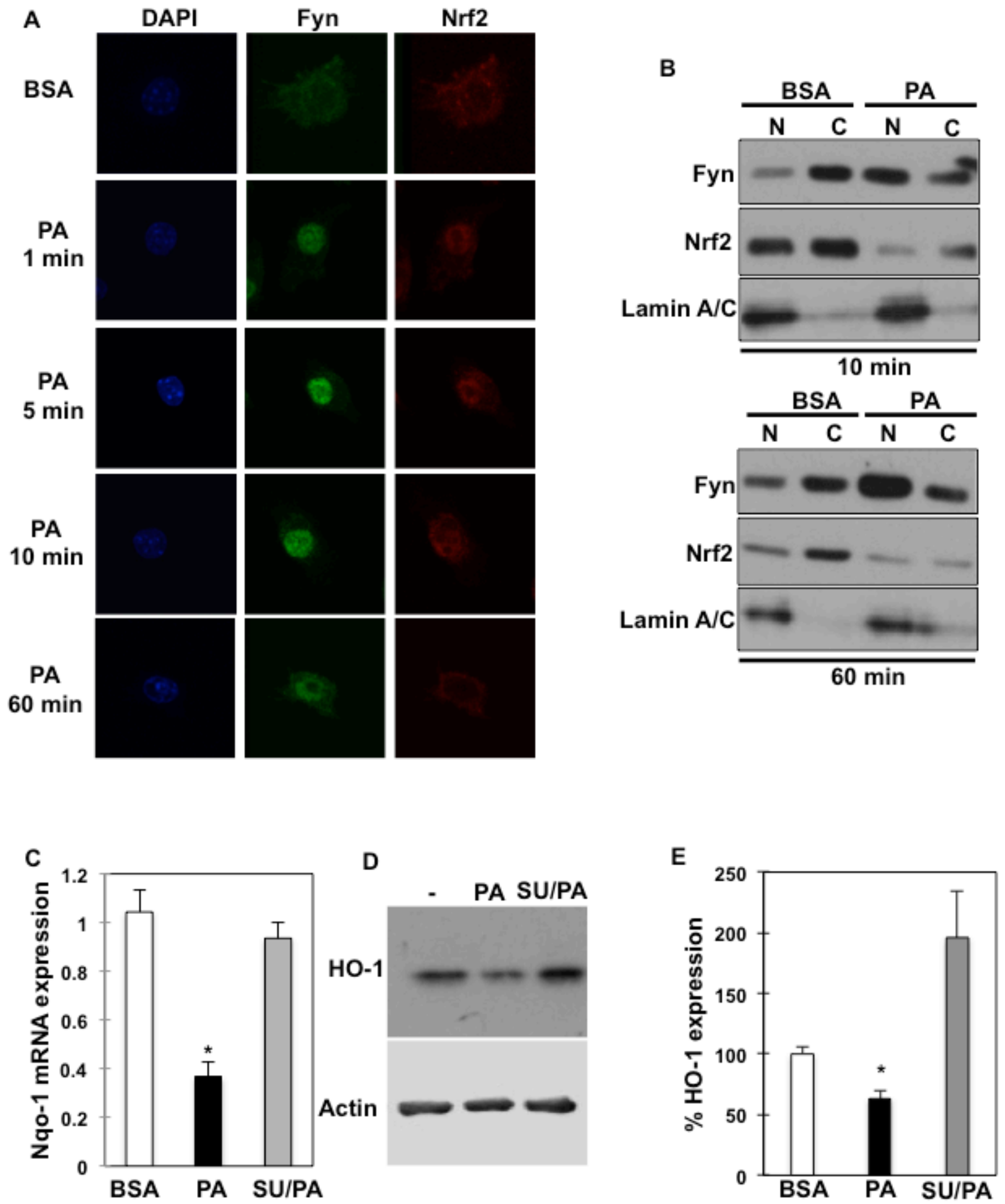

Figure 5: Fyn regulates Nrf2 subcellular localization and its downstream targets in response to palmitate. (A) RAW264.7 macrophages were incubated with BSA or $150 \mu \mathrm{M}$ PA for the indicated times (PA:BSA=2). Nuclei were visualized using DAPI staining, Fyn and Nrf2 subcellular localizations were assessed by immunofluorescence ( $\mathrm{n}=4$ experiments). (B) Cellular fractionation of RAW264.7 macrophages incubated with BSA, $150 \mu \mathrm{M}$ PA for 10 minutes or 60 min was performed (PA:BSA=2). Fyn and Nrf2 protein expressions were assessed in cytoplasmic and nuclear fractions. (C) Nqo-1 mRNA expression in RAW264.7 macrophages incubated with BSA, $150 \mu \mathrm{M}$ PA or $5 \mu \mathrm{M}$ SU6656 and $150 \mu \mathrm{M}$ PA (SU/PA), * $p<0.05(\mathrm{n}=4)$. (D) HO-1 protein expression in RAW264.7 macrophages in response to 150 $\mu \mathrm{M}$ PA or $5 \mu \mathrm{M}$ SU6656 and $150 \mu \mathrm{M}$ PA (SU/PA) (n=3 experiments). (E) Signal quantification of HO-1 protein expression in RAW264.7 cells corrected by actin and normalized to BSA condition. * $p<0.05(\mathrm{n}=3)$. 
SVF from low-fat fed mice (Figure 3A), as the mice are also exposed, although in lesser amount, to saturated fats in their diet.

\section{Saturated but not unsaturated FAs activate Fyn kinase in macrophages}

Palmitate-induced Fyn nuclear re-localization was blocked when cells were treated with the Fyn kinase inhibitor SU6656 (Figure 4A, panels g, h and Figure 4B), suggesting that Fyn kinase catalytic activity was required for palmitate-mediated Fyn nuclear localization. Supporting this, Fyn kinase activity was 6-fold higher in cells treated with palmitate, whilst it was much more moderately increased, if at all, after oleate (2-fold) or DHA (no increase) exposure (Figure 4C).

\section{Fyn inhibition blocks palmitate pro- inflammatory effects}

To investigate the physiological relevance of Fyn subcellular re-localization after palmitate exposure, and whether this affects macrophage activation in response to saturated fats, we next investigated palmitate effects on Nrf2 subcellular localization. Nrf2 is an important transcription factor that regulates anti-oxidative gene expression and is under Fyn regulatory control. Nrf2 localized in the cytoplasm of un-stimulated RAW264.7 macrophages (Figure 5A) as previously reported [28-30]. Time course experiments in RAW264.7 macrophages treated with palmitate revealed that both Fyn and Nrf2 accumulated in the nucleus after a short palmitate exposure (Figure 5A). However, while Fyn remained in the nucleus (Figure 5A, middle panels), the Nrf2 signal decreased in the nucleus with time (Figure 5A, right panels) and Nrf2 was cytosolic in approximately $75 \%$ of cells after 10 minutes (Figure 5A and Supplementary Figure 3). This was essentially confirmed by subcellular fractionations of RAW264.7 cells (Figure 5B). Notably, $\mathrm{Nrf} 2$ expression appeared slightly reduced after $10 \mathrm{~min}$ and is decreased after 60 min (Figure 5B, lower panel), which could be explained by increased Keap 1 expression and correlated increase in Nrf2 ubiquitination levels detected at $10 \mathrm{~min}$ and robustly observed after $30 \mathrm{~min}$ (Supplementary Figure 4). To evaluate Nrf2 functionality after palmitate exposure, Nrf2 downstream targets $\mathrm{NAD}(\mathrm{P}) \mathrm{H}$ dehydrogenase [quinone]-1 (Nqo-1) and Heme oxygenase-1 (HO-1) mRNA and protein expression were determined in RAW264.7 cells. Consistently with Nrf2 cytosolic localization, Nqo-1 mRNA expression was reduced after palmitate treatment. Importantly, Nqo-1 expression was rescued after Fyn inhibition with SU6656 (Figure 5C). Similarly, HO-1 protein expression was reduced after palmitate treatment and rescued after Fyn inhibition with SU6656 in RAW264.7 (Figure 5D, E) as well as in J774 (Supplementary Figure 5) macrophage models.

\section{DISCUSSION}

Obesity is a major worldwide health dilemma aggravated by refined diets rich in fats. Obesity is characterized by chronic low-grade inflammation and high fat diets promote invasion of immune cells, particularly macrophages into peripheral tissues. Recent studies have suggested that the number of macrophages [12] rather than their phenotype increases inflammation in obese individuals. This is also supported by the recent observation that M1 cell-surface markers are lacking in ATMs of obese humans and mice [14], which demonstrates that the phenotypic switch from anti-inflammatory (M2) to pro-inflammatory (M1) macrophages, and its role in adipose tissue inflammation is more complex than originally described. However, Kratz et al. [14] also showed that the saturated FA palmitate stimulates the macrophage population to resemble the M1 phenotype, e.g. by having an increased lipogenic profile. This strongly supports the concept that high-fat diets participate in the modification of the macrophage activation in peripheral tissues. However, not all fats are equal. Indeed, saturated fatty acids (FAs) increase pro-inflammatory gene expression, cytokine expression and reactive oxygen species (ROS) production in macrophages [31,32] whilst unsaturated, particularly n-3 polyunsaturated FAs (PUFAs) reduce pro-inflammatory processes in macrophages [33]. Whilst the effects of FAs on metabolic cascades (e.g. insulin signaling) have been largely studied, the role of these nutrients and how they modulate immune function is still unclear. Particularly, the molecular links (or "sensors") relaying FA effects on the macrophage function are still unknown. Therefore, molecules linking immune function and energy are of special interest. Previously, we showed that Fyn is a regulator of whole body lipid metabolism [24]. Remarkably, Fyn is also a major regulator of innate immune function, participating in TollLike Receptor (TLR) signaling in macrophages as well as in T-cell receptor signaling in T cells [34]. Additionally, Fyn interacts with the FA transporter CD36 [35, 36], which expression is greatly induced in palmitate-treated macrophages [14]. Therefore, we hypothesized that Fyn may transduce the inflammatory effects of saturated FAs. Consistently, we determined that Fyn kinase activity was greatly increased in response to palmitate; and palmitate pro-inflammatory effects were blunted in BMDMs lacking Fyn. Interestingly, PUFAs such as DHA were unable to activate Fyn; and Fyn was not re-localized to the nucleus in response to DHA (Figure 3 and Figure 4). This suggested that Fyn activation might be part of a signaling cascade distinguishing between the effects of saturated and unsaturated FAs on macrophage function. Further studies are necessary to identify mechanisms by which saturated 
and unsaturated FAs have divergent effects of Fyn kinase activation. It is suggested that Fyn distributes diffusely throughout the plasma membrane in some macrophage models and under particular stimuli, Fyn may undergo endocytosis [37]. As such, one could argue that saturated but not unsaturated FAs might trigger such mechanism to induce Fyn re-localization. It is known that CD36 and Fyn kinase interact [35], thus it is possible that mechanisms leading to palmitate-dependent Fyn activation might be mediated by CD36, which is induced by palmitate in macrophages. Nonetheless, the molecular mechanism of activation remains to be investigated and was beyond the scope of our study.

We found that, following palmitate exposure, Fyn rapidly re-localized into the nucleus of macrophages. This was subsequently followed by Nrf2 nuclear exclusion. Interestingly, Nrf2 action is also regulated by high fat diets; and FA-dependent regulation of Nrf2 appears to be dependent on the type of dietary fat [38], i.e. saturated fats inhibit Nrf2 signaling whereas unsaturated fats activate this pathway [20]. Therefore, it appears that FAs regulate Nrf2 function in an opposite pattern to that through which they regulate Fyn activity. This is consistent with the fact that Fyn exerts an inhibitory action on Nrf2. Our data and those of others showed that under basal conditions, Nrf2 localized in the cytoplasm of cells [28-30]. However, Nrf2 quickly relocalized to the nucleus after palmitate exposure, along with Fyn kinase. This might appear counter-intuitive since Fyn inhibits Nrf2 action by promoting Nrf2 nuclear exclusion. However, we observed that after this first phase, the Nrf2 nuclear signal gradually decreased. As Fyn remained in the nucleus, this suggested that Nrf2 function was inhibited as a consequence of the persistent nuclear localization of Fyn. In line with this, we observed a decrease in the expression of downstream targets of Nrf2 after palmitate exposure. Mechanisms leading to Nrf2 initial nuclear localization in response to palmitate remain to be elucidated. However, it appears reasonable to suggest that this may be a mechanism to protect the cells from the deleterious effects of ROS production. Supporting this, we observed that expression of the Nrf2 targets Nqo-1 and HO-1 were transiently elevated shortly after palmitate exposure (data not shown). This early phase was then inhibited by the persistent presence of Fyn in the nucleus, which triggers Nrf2 nuclear exclusion and cytosolic degradation.

Importantly, the effects of palmitate on Fyn nuclear re-localization were blocked when cells were incubated with SU6656 (a Fyn inhibitor), and palmitate-mediated inhibition of Nrf2 pathway was lifted. SU6656 is a potent Fyn inhibitor but it is also known to inhibit other Src kinase family members. Yet, SU6656 reduced palmitateinduced ROS production in RAW264.7 but had no effect in shFyn-RAW264.7 cells (data not shown). This strongly supported that SU6656 effects were most likely through
Fyn specific inhibition. Lastly, SU6656 was recently shown to activate AMPK [39] and we have demonstrated that Fyn inhibition itself activates AMPK in peripheral tissues [24]. Therefore, we cannot exclude that the inhibitory action of SU6656 on palmitate downstream effects may be through Fyn inhibition combined with AMPK activation. Further examinations would be necessary to dissect this possibility but, in any case, the ensemble of our data support that Fyn kinase activity is necessary for the palmitate-dependent inhibition of Nrf2 pathway.

Dietary fats are well-established modulators of macrophage function. However, molecular events linking FAs to macrophage activation are not completely identified. Building on our present data, we provide a novel mechanism by which macrophages respond differentially to saturated FAs than to unsaturated FAs. This occurs through the selective activation of Fyn kinase by saturated FAs, and its antagonism by unsaturated FAs. This establishes Fyn as an integral component of a signaling pathway able to sense dietary cues that contribute to the establishment of inflammation in nutritional obesity. Further studies are now necessary to identify the upstream regulatory mechanisms by which saturated and unsaturated FAs differentially regulate Fyn kinase activity; and how Fyn is successively transported to the nucleus of macrophages.

\section{MATERIALS AND METHODS}

\section{Reagents}

Rabbit polyclonal Fyn antibody (FYN3), Heme Oxygenase-1, Nrf2 (H300), goat polyclonal Nrf2 (T19) and Nrf2 (C20) antibodies were from Santa Cruz Biotechnology (Santa Cruz, CA, USA). GAPDH antibody was from MBL international (Woburn, MA, USA). LaminA/C and P115 antibodies were from Cell Signaling Technology (Danvers, MA, USA).

\section{Animals}

Mice homogenous for the $F y n^{\text {tm } 1 \text { Sor }}$ targeted mutation (129-Fyn tmlSor $\left.^{\mathrm{J}}\right)($ fynKO) and their controls (129S1/SvImJ) were obtained from the Jackson Laboratory (Bar Harbor, ME, USA) and housed in a facility equipped with a $12 \mathrm{~h}$ light/dark cycle. 8 weeks old male mice were fed either a standard chow diet (Research Diets, New Brunswick, NJ, USA) containing $70 \%$ (Kcal) carbohydrates, $20 \%$ protein, and $10 \%$ fat or a high fat diet containing 20\% (Kcal) carbohydrates, 20\% protein and $60 \%$ fat for 8 weeks. Composition of the diets D12392 (60\% fat) and D12450B (control diet) can be found at http://www.researchdiets.com/opensource-diets/ stock-diets. Essentially, saturated fats were increased by $10 \%$ and polyunsaturated fats were reduced by $15 \%$ in D12492 compared to the control diet. All studies were 
approved by and performed in compliance with the guidelines of the Yeshiva University Institutional Animal Care and Use Committee (IACUC).

\section{Bone marrow derived macrophages (BMDM) isolation and differentiation}

BMDMs from control and fynKO mice were prepared using a standard method. Briefly, marrow was flushed from the femurs using DMEM media with $10 \%$ FBS. The suspension was filtered through a $70 \mu \mathrm{m}$ cell strainer and red cells were removed. The remaining precursor cells were plated and differentiated into BMDMs with macrophage colony-stimulating factor (M-CSF, $10 \mathrm{ng} / \mathrm{ml}$ ) (R\&D Systems, Minneapolis, MN, USA). Differentiation was typically achieved within 6 days.

\section{Stromal vascular fraction (SVF) cells isolation}

Minced adipose tissue samples were treated with $0.05 \mathrm{mg} / \mathrm{mL}$ Liberase (TM Research Grade; Roche Applied Science, Indianapolis, IN) and incubated at $37^{\circ} \mathrm{C}$ for $20 \mathrm{~min}$. Samples were passed through a sterile $250 \mathrm{~mm}$ nylon mesh. The suspension was centrifuged at $1,000 \mathrm{xg}$ for $5 \mathrm{~min}$. The precipitated cells (SVF) were re-suspended in erythrocyte lysis buffer. The erythrocyte-depleted SVF cells were centrifuged at $500 \mathrm{xg}$ for $5 \mathrm{~min}$ and resuspended in DMEM medium supplemented with $10 \%$ FCS and $1 \%$ Penicillin/Streptomycin.

\section{Macrophages isolation}

SVF from lean and fat-fed mice $(\mathrm{n}=5$ for the fatfed mice and $n=8$ for the control mice) were prepared as described above. SVF were treated with erythrocyte lysis buffer and washed two times in PBS. Positive selection to enrich for F4/80 cells was performed using the MagniSort Mouse F4/80 positive selection kit (eBioscience, Inc,). Briefly, $10^{8}$ cells were suspended in a separation buffer and incubated with the selection antibody for $60 \mathrm{~min}$. Cells were washed and selection beads were added to the suspension. Following sorting, cells were washed and protein homogenates were immediately prepared.

\section{Fatty acid preparation and incubation}

Palmitic acid (PA) (Sigma, St. Louis, MO, USA) 50 $\mathrm{mM}$ was prepared in methanol and stored at $-20^{\circ} \mathrm{C}$. On the day of use, indicated PA concentrations were prepared in fatty acid-free and low endotoxin BSA at a ratio FA: $\mathrm{BSA}=2$. Equal volumes of methanol (final concentration $<0.1 \%$ ) in fatty acid-free BSA were applied to control cells. Where Fyn pharmacological inhibitor was used, cells were treated with $5 \mu \mathrm{M}$ SU6656 (Calbiochem, San Diego, CA, USA) for $60 \mathrm{~min}$.

\section{Fyn tyrosine activity}

Activity was determined with a Universal Tyrosine Kinase activity assay kit (Takara Bio Inc., Shiga, Japan). Cells were homogenized in a NP40- based buffer. Homogenates were centrifuged for $15 \mathrm{~min}$ at $14,000 \mathrm{xg}$ at $4{ }^{\circ} \mathrm{C}$ and supernatants were collected. Whole cell lysates $(0.5-1 \mathrm{mg})$ were incubated with $4 \mu \mathrm{g}$ of FYN3 rabbit polyclonal antibody coupled with Catch and Release columns (Millipore, Billerica, MA, USA) for $2 \mathrm{~h}$ at $4^{\circ} \mathrm{C}$. Immuno-complexes were diluted and samples were mixed with an ATP solution before being incubated in an ELISA plate. Kinase activity was corrected by the protein concentration.

\section{Quantitative PCR analysis}

Cells were homogenized into QIAzol Lysis Reagent. Total RNA was isolated using RNeasy ${ }^{\circledR}$ Mini Kit (Qiagen Sciences, Maryland, USA) and reversetranscribed to cDNA using the SuperScript VILO cDNA synthesis kit (Invitrogen, Carlsbad, CA, USA). TaqMan (Applied Biosystems, Branchburg, NJ, USA) RT-PCR was performed for measurement of mRNA of Tumor Necrosis Factor-alpha (TNF-a) (Mm00443260_g1), Interleukin-6 (IL-6) (Mm00446190_m1), Nitric oxide synthase-2 (Nos2) (Mm00440502_m1), Fyn (Mm00433373_m1), Heme Oxygenase-1 (HO-1) (Mm00516005_m1), NAD(P)H dehydrogenase [quinone]-1 (Nqo-1) (Mm01253561_m1), and $N A D(P) H$ Oxidase. Relative expression levels of the mRNAs were determined using standard curves using the Applied Biosystems 7900HT Sequence Detection System from Applied Biosystems. Samples were adjusted for total mRNA content by comparison with Rpl7 expression. All primer-probe mixtures were from Applied Biosystems (Branchburg, NJ, USA).

\section{Western blotting}

Cells were homogenized in a NP-40 lysis buffer containing protease and phosphatase inhibitors and protein concentration was determined using the $\mathrm{BCA}$ method. Homogenates were centrifuged for $15 \mathrm{~min}$ at $13,000 \mathrm{xg}$ at $4{ }^{\circ} \mathrm{C}$ and supernatants were collected. Protein samples $(40 \mu \mathrm{g})$ were separated onto 8 or $10 \%$ reducing polyacrylamide gels and electroblotted onto Immobilon-P polyvinylidene difluoride membranes (BioRad Laboratories, Hercules, CA, USA). Immunoblots were blocked either 5\% milk in Tris-buffered saline or with Blocking Buffer for Fluorescent Western Blotting (Rockland Antibodies \& Assays, Gilbertsville, PA, USA) for $2 \mathrm{~h}$ at room temperature and incubated overnight at $4^{\circ} \mathrm{C}$ with the indicated antibodies in Tris-buffered saline and $0.05 \%$ Tween 20 (TBST) containing 1\% BSA. Membranes were washed in TBST and incubated with horseradish peroxidase-conjugated secondary antibodies $(1: 30,000)$ for $45 \mathrm{~min}$ at room temperature. Membranes 
were washed in TBST, and antigen-antibody complexes were visualized by chemiluminescence using an ECL kit (Pierce, Rockford, IL, USA). Alternatively, immunoblots were incubated with IRDye800CW Goat Anti Mouse $(\mathrm{H}+\mathrm{L})$ or IRDye680 Goat Anti Rabbit $(\mathrm{H}+\mathrm{L})$ secondary antibodies and signal was detected with the Odyssey $\mathbb{R}$ Infrared Imaging System (Li-COR Biotechnology, Lincoln, NE, USA). The relative band intensity was quantified using the ImageJ software.

\section{Nuclear and cytoplasmic protein fractions}

Cells were treated as indicated and nuclear and cytoplasm extracts were prepared using a kit from Pierce (Rockford, IL, USA). Proteins were separated onto 10\% reducing polyacrylamide gels and electroblotted onto Immobilon-P polyvinylidene difluoride membranes. Immunoblots were processed as described above. Antigen-antibody complexes were visualized by chemiluminescence or using the Odyssey ${ }^{\circledR}$ Infrared Imaging System, as above.

\section{Cell culture and silencing}

The human embryonic kidney cells (HEK-293T), the mouse macrophage cell lines RAW264.7 (ATCC TIB-71) and J774 (ATCC TIB-67) were obtained from ATCC (Manassas, VA, USA) and maintained in DMEM supplemented with $10 \%$ fetal calf serum and $1 \%$ penicillin/streptomycin at $37^{\circ} \mathrm{C}$ in a humidified incubator with 5\% CO2. Fyn kinase-silenced and control stable cell lines were produced by transducing the macrophage cell lines with either MISSION shRNA lentivirus Fyn particles (TRCN000023380) or MISSION non-mammalian target shRNA control particles (SHC002) (Sigma). The transduced cells were selected with $3 \mu \mathrm{g} / \mathrm{ml}$ Puromycin for 10 days.

Transient transfections of HEK293 cells were performed using the FugeneHD (Roche Applied Science, Basel, Switzerland) according to the manufacturer's protocol.

\section{Immunofluorescence}

Cells were seeded in 8 wells-chamber slides and stimulated with PA, oleate, DHA or SU6656. After the indicated incubation time, cells were washed with PBS and fixed for $30 \mathrm{~min}$ in PBS containing 4\% paraformaldehyde and $2 \%$ sucrose, followed by three washes in PBS. Slides were incubated in PBS with Triton-X-100 (PBST) for $5 \mathrm{~min}$ at $4{ }^{\circ} \mathrm{C}$ to permeabilize the cells. Cells were washed and blocked in PBST with BSA $5 \%$ for $1 \mathrm{~h}$ at room temperature and incubated with either Fyn or Nrf2 antibody diluted 1:200 in PBST-BSA $2 \%$, followed by Alexa Fluor 488 anti-rabbit IgG or IgG or Alexa Fluor 594 anti-goat IgG for $1 \mathrm{~h}$ at room temperature. Samples were mounted with Prolong Gold anti-fade reagent with DAPI (Life Technologies). Fluorescence images were acquired using a confocal fluorescence microscope (TCS SP5 confocal; Leica microsystems, Buffalo Grove, IL, USA).

\section{Cellular reactive oxygen species detection assay}

Cells were seeded onto 96 wells plate and preloaded with $10 \mu \mathrm{M} \quad 2^{\prime}, 7^{\prime}$-dichlorodihydrofluorescein diacetate (H2DCFDA) (Life Technologies) at $37^{\circ} \mathrm{C}$ for $60 \mathrm{~min}$ at room temperature. Cells were PBS rinsed (2 times) and reactive oxygen species (ROS) production was assessed by quantifying the fluorescence of the cells after PA exposure (10 min) using the BioTek Synergy 2 plate reader (BioTek Instruments, Winooski, VT, USA).

\section{Statistics}

Results are expressed as mean \pm standard error of the mean (s.e.m). The data were analyzed by one-way ANOVA followed by post hoc analysis for comparisons between individual groups when group numbers were $>2$. Differences were considered statistically significant at a level of $p<0.05$. Differences between 2 treatments were tested for statistical significance $(p<0.05)$ using a Student's unpaired t-test.

\section{Abbreviations}

KO: knockout; WT: wild type; BMDM: bone marrow derived macrophages; HFD: high fat diet; Nrf2: nuclear-factor erythroid2-related factor2; AMPK: AMPactivated protein kinase; DHA: docosahexaenoic acid; PA: palmitate; SVF: stromal vascular fraction; ROS: reactive oxygen species.

\section{Author contributions}

Original idea, experimental conception, manuscript writing and editing (CCB); Experimental execution, writing and editing (ET; MV, CCB); Experimental execution (TWAL, EY); writing and editing (VAZ, JEP).

\section{ACKNOWLEDGMENTS}

The authors wish to thank the members of the Diabetes Center at Albert Einstein College of Medicine for their continuous support.

\section{CONFLICTS OF INTEREST}

The authors do not have any conflicts of interest. 


\section{FUNDING}

This work was supported by the National Institutes of Health (DK81412 (CCB)) and a Research \& Development Strategic Award from the University of Warwick.

\section{REFERENCES}

1. Finucane MM, Stevens GA, Cowan MJ, Danaei G, Lin JK, Paciorek CJ, Singh GM, Gutierrez HR, Lu Y, Bahalim AN, Farzadfar F, Riley LM, Ezzati M. National, regional, and global trends in body-mass index since 1980: systematic analysis of health examination surveys and epidemiological studies with 960 country-years and 9.1 million participants. Lancet. 2011; 377:557-567.

2. Xu H, Barnes GT, Yang Q, Tan G, Yang D, Chou CJ, Sole J, Nichols A, Ross JS, Tartaglia LA, Chen H. Chronic inflammation in fat plays a crucial role in the development of obesity-related insulin resistance. J Clin Invest. 2003; 112:1821-1830

3. Weisberg SP, McCann D, Desai M, Rosenbaum M, Leibel RL, Ferrante AW Jr. Obesity is associated with macrophage accumulation in adipose tissue. J Clin Invest. 2003; 112:1796-1808.

4. Fink LN, Costford SR, Lee YS, Jensen TE, Bilan PJ, Oberbach A, Bluher M, Olefsky JM, Sams A, Klip A. Proinflammatory macrophages increase in skeletal muscle of high fat-fed mice and correlate with metabolic risk markers in humans. Obesity (Silver Spring). 2014; 22:747-757.

5. Morinaga $\mathrm{H}$, Mayoral $\mathrm{R}$, Heinrichsdorff $\mathrm{J}$, Osborn O, Franck N, Hah N, Walenta E, Bandyopadhyay G, Pessentheiner AR, Chi TJ, Chung H, Bogner-Strauss JG, Evans RM, et al. Characterization of distinct subpopulations of hepatic macrophages in HFD/obese mice. Diabetes. 2015; 64:1120-1130.

6. Winer DA, Winer S, Shen L, Wadia PP, Yantha J, Paltser G, Tsui H, Wu P, Davidson MG, Alonso MN, Leong HX, Glassford A, Caimol M, et al. B cells promote insulin resistance through modulation of $\mathrm{T}$ cells and production of pathogenic IgG antibodies. Nat Med. 2011; 17:610-617.

7. Nishimura S, Manabe I, Nagasaki M, Eto K, Yamashita H, Ohsugi M, Otsu M, Hara K, Ueki K, Sugiura S, Yoshimura K, Kadowaki T, Nagai R. CD8+ effector T cells contribute to macrophage recruitment and adipose tissue inflammation in obesity. Nat Med. 2009; 15:914-920.

8. Talukdar S, Oh da Y, Bandyopadhyay G, Li D, Xu J, McNelis J, Lu M, Li P, Yan Q, Zhu Y, Ofrecio J, Lin M, Brenner MB, Olefsky JM. Neutrophils mediate insulin resistance in mice fed a high-fat diet through secreted elastase. Nat Med. 2012; 18:1407-1412.

9. Liu J, Divoux A, Sun J, Zhang J, Clement K, Glickman JN, Sukhova GK, Wolters PJ, Du J, Gorgun CZ, Doria A, Libby P, Blumberg RS, et al. Genetic deficiency and pharmacological stabilization of mast cells reduce diet-induced obesity and diabetes in mice. Nat Med. 2009; 15:940-945.

10. Lumeng CN, Deyoung SM, Bodzin JL, Saltiel AR. Increased inflammatory properties of adipose tissue macrophages recruited during diet-induced obesity. Diabetes. 2007; 56:16-23.

11. Shapiro H, Lutaty A, Ariel A. Macrophages, meta-inflammation, and immuno-metabolism. ScientificWorldJournal. 2012; 11:2509-2529.

12. Xu X, Grijalva A, Skowronski A, van Eijk M, Serlie MJ, Ferrante AW Jr. Obesity activates a program of lysosomaldependent lipid metabolism in adipose tissue macrophages independently of classic activation. Cell Metab. 2013; 18:816-830.

13. Lumeng CN, Bodzin JL, Saltiel AR. Obesity induces a phenotypic switch in adipose tissue macrophage polarization. J Clin Invest. 2007; 117:175-184.

14. Kratz M, Coats BR, Hisert KB, Hagman D, Mutskov V, Peris E, Schoenfelt KQ, Kuzma JN, Larson I, Billing PS, Landerholm RW, Crouthamel M, Gozal D, et al. Metabolic dysfunction drives a mechanistically distinct proinflammatory phenotype in adipose tissue macrophages. Cell Metab. 2014; 20:614-625.

15. Xue B, Yang Z, Wang X, Shi H. Omega-3 polyunsaturated fatty acids antagonize macrophage inflammation via activation of AMPK/SIRT1 pathway. PLoS One. 2012; 7:e45990.

16. Chan KL, Pillon NJ, Sivaloganathan DM, Costford SR, Liu Z, Theret M, Chazaud B, Klip A. Palmitoleate reverses high fat-induced proinflammatory macrophage polarization via AMP-activated protein kinase (AMPK). J Biol Chem. 2015; 290:16979-16988.

17. Mounier R, Theret M, Arnold L, Cuvellier S, Bultot L, Goransson O, Sanz N, Ferry A, Sakamoto K, Foretz M, Viollet B, Chazaud B. AMPKalpha1 regulates macrophage skewing at the time of resolution of inflammation during skeletal muscle regeneration. Cell Metab. 2013; 18:251-264.

18. Yang Z, Wang X, He Y, Qi L, Yu L, Xue B, Shi H. The full capacity of AICAR to reduce obesity-induced inflammation and insulin resistance requires myeloid SIRT1. PLoS One. 2012; 7:e49935.

19. Pi J, Leung L, Xue P, Wang W, Hou Y, Liu D, YehudaShnaidman E, Lee C, Lau J, Kurtz TW, Chan JY. Deficiency in the nuclear factor E2-related factor-2 transcription factor results in impaired adipogenesis and protects against dietinduced obesity. J Biol Chem. 2010; 285:9292-9300.

20. Tanaka Y, Ikeda T, Yamamoto K, Ogawa H, Kamisako T. Dysregulated expression of fatty acid oxidation enzymes and iron-regulatory genes in livers of Nrf2-null mice. J Gastroenterol Hepatol. 2012; 27:1711-7.

21. Jain AK, Jaiswal AK. Phosphorylation of tyrosine 568 controls nuclear export of Nrf2. J Biol Chem. 2006; 281:12132-12142. 
22. Jain AK, Jaiswal AK. GSK-3beta acts upstream of Fyn kinase in regulation of nuclear export and degradation of NF-E2 related factor 2. J Biol Chem. 2007; 282:16502-16510.

23. Kaspar JW, Jaiswal AK. Tyrosine phosphorylation controls nuclear export of Fyn, allowing Nrf2 activation of cytoprotective gene expression. FASEB J. 2011; 25:1076-1087.

24. Bastie CC, Zong H, Xu J, Busa B, Judex S, Kurland IJ, Pessin JE. Integrative metabolic regulation of peripheral tissue fatty acid oxidation by the SRC kinase family member Fyn. Cell Metab. 2007; 5:371-381.

25. Yamada E, Pessin JE, Kurland IJ, Schwartz GJ, Bastie CC. Fyn-dependent regulation of energy expenditure and body weight is mediated by tyrosine phosphorylation of LKB1. Cell Metab. 2010; 11:113-124.

26. Lee TW, Kwon H, Zong H, Yamada E, Vatish M, Pessin JE, Bastie CC. Fyn deficiency promotes a preferential increase in subcutaneous adipose tissue mass and decreased visceral adipose tissue inflammation. Diabetes. 2013; 62:1537-15346.

27. Panicker N, Saminathan H, Jin H, Neal M, Harischandra DS, Gordon R, Kanthasamy K, Lawana V, Sarkar S, Luo J, Anantharam V, Kanthasamy AG, Kanthasamy A. Fyn kinase regulates microglial neuroinflammatory responses in cell culture and animal models of Parkinson's disease. J Neurosci. 2015; 35:10058-10077.

28. Kobayashi A, Ohta T, Yamamoto M. Unique function of the Nrf2-Keap1 pathway in the inducible expression of antioxidant and detoxifying enzymes. Methods Enzymol. 2004; 378:273-286.

29. Cullinan SB, Gordan JD, Jin J, Harper JW, Diehl JA. The Keap1-BTB protein is an adaptor that bridges Nrf2 to a Cul3-based E3 ligase: oxidative stress sensing by a Cul3Keap1 ligase. Mol Cell Biol. 2004; 24:8477-8486.

30. Zhang DD, Lo SC, Cross JV, Templeton DJ, Hannink M. Keap 1 is a redox-regulated substrate adaptor protein for a Cul3-dependent ubiquitin ligase complex. Mol Cell Biol. 2004; 24:10941-10953.
31. Shi H, Kokoeva MV, Inouye K, Tzameli I, Yin H, Flier JS. TLR4 links innate immunity and fatty acid-induced insulin resistance. J Clin Invest. 2006; 116:3015-3025.

32. Kewalramani G, Fink LN, Asadi F, Klip A. Palmitateactivated macrophages confer insulin resistance to muscle cells by a mechanism involving protein kinase $\mathrm{C}$ theta and epsilon. PLoS One. 2011; 6:e26947.

33. Titos E, Rius B, Gonzalez-Periz A, Lopez-Vicario C, Moran-Salvador E, Martinez-Clemente M, Arroyo V, Claria J. Resolvin D1 and its precursor docosahexaenoic acid promote resolution of adipose tissue inflammation by eliciting macrophage polarization toward an M2-like phenotype. J Immunol. 2011; 187:5408-5418.

34. Salmond RJ, Filby A, Qureshi I, Caserta S, Zamoyska R. T-cell receptor proximal signaling via the Src-family kinases, Lck and Fyn, influences T-cell activation, differentiation, and tolerance. Immunol Rev. 2009; 228:9-22.

35. Huang MM, Bolen JB, Barnwell JW, Shattil SJ, Brugge JS. Membrane glycoprotein IV (CD36) is physically associated with the Fyn, Lyn, and Yes protein-tyrosine kinases in human platelets. Proc Natl Acad Sci U S A. 1991; 88:7844-7848.

36. Bull HA, Brickell PM, Dowd PM. Src-related protein tyrosine kinases are physically associated with the surface antigen CD36 in human dermal microvascular endothelial cells. FEBS Lett. 1994; 351:41-44.

37. Dwyer AR, Mouchemore KA, Steer JH, Sunderland AJ, Sampaio NG, Greenland EL, Joyce DA, Pixley FJ. Src family kinase expression and subcellular localization in macrophages: implications for their role in CSF-1-induced macrophage migration. J Leukoc Biol. 2016; 100:163-175.

38. Wang H, Khor TO, Saw CL, Lin W, Wu T, Huang Y, Kong AN. Role of Nrf2 in suppressing LPS-induced inflammation in mouse peritoneal macrophages by polyunsaturated fatty acids docosahexaenoic acid and eicosapentaenoic acid. Mol Pharm. 2010; 7:2185-2193.

39. Ross FA, Hawley SA, Auciello FR, Gowans GJ, Atrih A, Lamont DJ, Hardie DG. Mechanisms of paradoxical activation of AMPK by the kinase inhibitors SU6656 and sorafenib. Cell Chem Biol. 2017; 24:813-824. 